\title{
DISCRIMINATION OF TWO SPECIES OF ORANGUTANS (PONGO SP.): A RAPID PROTOCOL FOR REHABILITATION CENTRES AND ZOOS
}

\author{
DYAH PERWITASARI-FARAJALLAH ${ }^{1,2^{*}}$ \\ ${ }^{1}$ Primate Research Center, Bogor Agricultural University, Bogor 16151 Indonesia \\ ${ }^{2}$ Department of Biology, Faculty of Mathematics and Natural Sciences, Bogor Agricultural \\ University, Kampus IPB Darmaga, Bogor, Indonesia
}

\begin{abstract}
Currently orangutans are found in widely fragmented and isolated populations. Sumatran orangutan is primarily found in northern Sumatra, and the Bornean orangutans is distributed in Central, West, and East Kalimantan, Sarawak and Sabah. The determination of intra- and inter-species variation between Bornean and Sumatran orangutans is been stated to be essential for both the management of orangutan reintroduction projects and the planning of conservation strategies to preserve the remaining wild populations. This study aimed to identify two species of Orangutans (Pongo sp.) by means of RFLP (Restriction Fragment Length Polymorphisms) analyses of mitochondrial DNA (mtDNA). An approximately $540 \mathrm{bp}$ single fragment of the ND5 gene near the 5'-region was PCR amplified for all samples tested. Digestion pattern for both $A l u \mathrm{I}$ and $M s e \mathrm{I}$ were different between two groups of ND5 fragments in this study. Present result showed a rapid protocol to identify these two species by means of RFLP (Restriction Fragment Length Polymorphism) analyses of mtDNA (mitochondrial DNA). This technique can be applied easily to rehabilitation centres and zoos to resolve species discrimination problem.
\end{abstract}

Key words: Orangutans, Pongo sp., Sumatra, Borneo, discrimination

\section{INTRODUCTION}

The endangered orangutans (Pongo sp.) in the wild are only found on the islands of Borneo and Sumatra. Now, the orangutan is listed as a CITES Appendix 1 Endangered Species (most endangered). Orangutans are taxonomically classified as two distinct subspecies, the Bornean (Pongo pygmaeus pygmaeus) and the Sumatran (Pongo pygmaeus abelii), based primarily on their distinctive morphological and behavioral

*Corresponding author: witafar@yahoo.com 
characteristics (Groves 1971). However, some authors have recently argued that the populations should be promoted to species status (Zhi et al. 1996; Xu \& Arnason 1996; Muir et al. 2000) because the differences between the two subspecies are more identical than those relatively recognized species e.g. chimpanzee (Pan troglodytes) vs. bonobo (Pan paniscus), horse (Equus caballus) vs. donkey (Equus asianus). These studies have been based on morphology (size, hair color, beard, size of cheek pads) and genetics (allozymes, nuclear RFLPs, mtDNA sequence, and chromosomal inversions) which seem to have correlations with the island of origin.

At present orangutans inhabit in a widely fragmented and isolated populations. While Sumatran orangutan is primarily found in northern Sumatra, and the Bornean is distributed in Central, West, and East Kalimantan, Sarawak and Sabah, they were never found in Brunei and South Kalimantan (Rijksen \& Meijaard 1999). The determination of intra- and inter-species variation between Bornean and Sumatran orangutans has been noted to be essential for both the management of orangutan in the reintroduction projects and the planning of conservation strategies for the remaining wild populations (Janczewski, Goldman \& O'Brien 1990; Uchida 1996).

This study aimed to identify two species of Orangutans (Pongo sp.) by means of RFLP (Restriction Fragment Length Polymorphisms) analyses of mitochondrial DNA (mtDNA).

\section{MATERIALS AND METHOD}

\section{Samples}

Blood samples are taken from orangutans being rehabilitated at the Rehabilitation Centers. Heparinized blood samples are stored at $-20^{\circ} \mathrm{C}$ until used. There are $20 \mathrm{blood}$ samples used, 18 of which were originated in Borneo and the two were from Sumatra.

\section{DNA preparation}

Blood samples will be subjected to QIAGEN DNA blood extraction kit using the manufacturer's suggested protocol.

\section{General PCR and RFLP methods}

Polymerase Chain Reaction (PCR). Whole genomic DNA extracts were used for PCR reactions. PCR reaction mixtures contain $1 \mathrm{mM} \mathrm{MgCl}_{2}$, 1x Taq buffer, $0.2 \mathrm{mM}$ primers, $1 \mathrm{U}$ Taq polymerase (PROMEGA). PCR was performed under the conditions of Zhang et al (2001): $94^{\circ} \mathrm{C} 40$ s, $56{ }^{\circ} \mathrm{C} 40$ s, $72^{\circ} \mathrm{C} 30$ s, for 35 cycles, with 94 ${ }^{\circ} \mathrm{C} 12 \mathrm{~min}$ at the beginning and $72^{\circ} \mathrm{C}, 10 \mathrm{~min}$ at the end. ND5 region of mitochondrial DNA were amplified using primers of

ND5f (forward) 5'-TAA-CCG-CCC-TCA-CCT-TAA-CT'T-CCC-3' (24 bp)

ND5r (reverse) 5'-GGT-CAG-GAT-GAA-GCC-AAT-GTC-G-3' (22 bp)

Restriction Fragment Length Polymorphisms (RFLP). PCR products were digested overnight by using restriction enzymes (Table 1 ) at $37^{\circ} \mathrm{C}$. 
Discrimination of two species of Orangutans (Pongo sp.) - Dyah Perwitasari.

Table 1. Restriction Enzymes used for PCR-RFLP analyses

\begin{tabular}{ccc}
\hline No. & Restriction Enzymes & Recognition Sites \\
\hline 1. & AluI & AGCT \\
2. & Hha $\mathrm{I}$ & GCGC \\
3. & $M s e \mathrm{I}$ & TTAA \\
4. & Sau3A & GATC \\
\hline
\end{tabular}

The digested products were separated on 5-6\% PAGE in TBE buffer then stained with silver. Fragment sizes were determined using a 100 bp DNA ladder marker (BioRad).

\section{RESULTS AND DISCUSSION}

An approximately 540 bp single fragment of the ND5 gene near the 5'-region was PCR amplified for all samples tested (Fig. 1).

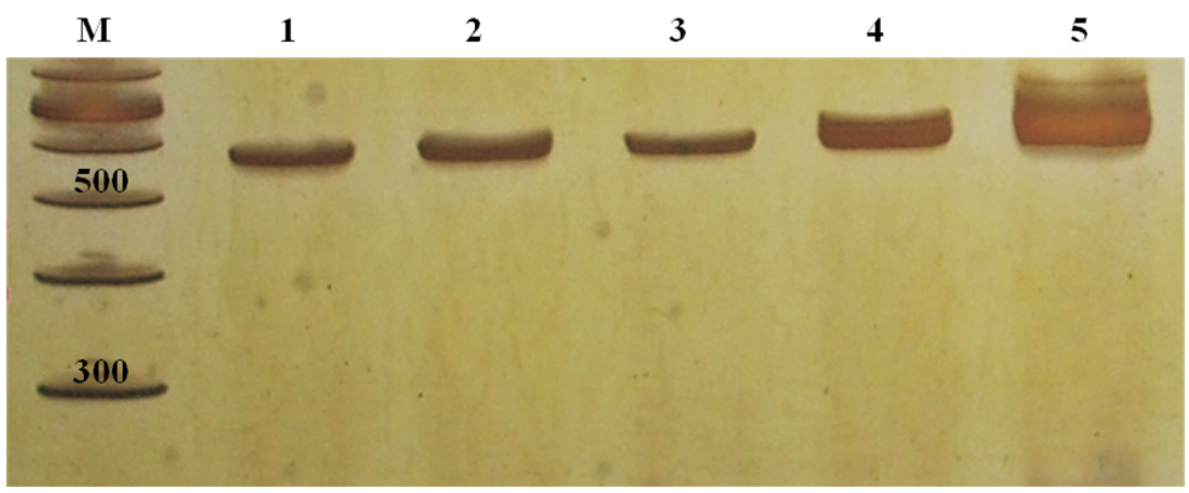

Figure 1. ND5 gene fragments of mtDNA amplified using primers reported by Zhang et al (2001); fragment size +540 bp; M: DNA ladder marker; 1-5: representative samples

Using reference sequences from DNA database for Sumatran and Bornean species (Genbank accession numbers are AF255448-449, AF255450-452, AF255454), the most appropriate restriction enzymes that indicated a remarkable differences among squences of two species of Orangutan were determined. Among four restriction enzymes restriction enzymes ( $A l u \mathrm{I}, H h a \mathrm{I}, \mathrm{Mse} \mathrm{I}$ and $S a u 3 \mathrm{~A})$, two restriction enzymes were selected for RFLP (restriction fragment length polymorphisms) analyses, these are $A l u \mathrm{I}$ and $M s e \mathrm{I}$ (Table 2). Digestion result using $A l u \mathrm{I}$ yielded two fragments of approximately 220 and 320 bp respectively for Bornean and three fragments of 50, 220 and $270 \mathrm{bp}$ for Sumatran orangutans. Furthermore MseI digested the PCR product into two fragments of 220 and $320 \mathrm{bp}$ for Bornean and two fragments of 50 and 490 bp for Sumatran orangutans. Hence although Bornean and Sumatran orangutans revealed two fragments, their sizes were different. Observed numbers of DNA fragments after enzymatic digestion is summarized in Table 3. 
Table 2. Reference sequences from DNA database are given with Pa (Sumatran species) and Pp (Bornean species) letters. Recognition sites of MseI (TTAA), AluI (AGCT) and Sau3A (GATC) are indicated with underlines; No recognition sites for $\mathrm{HhaI}$

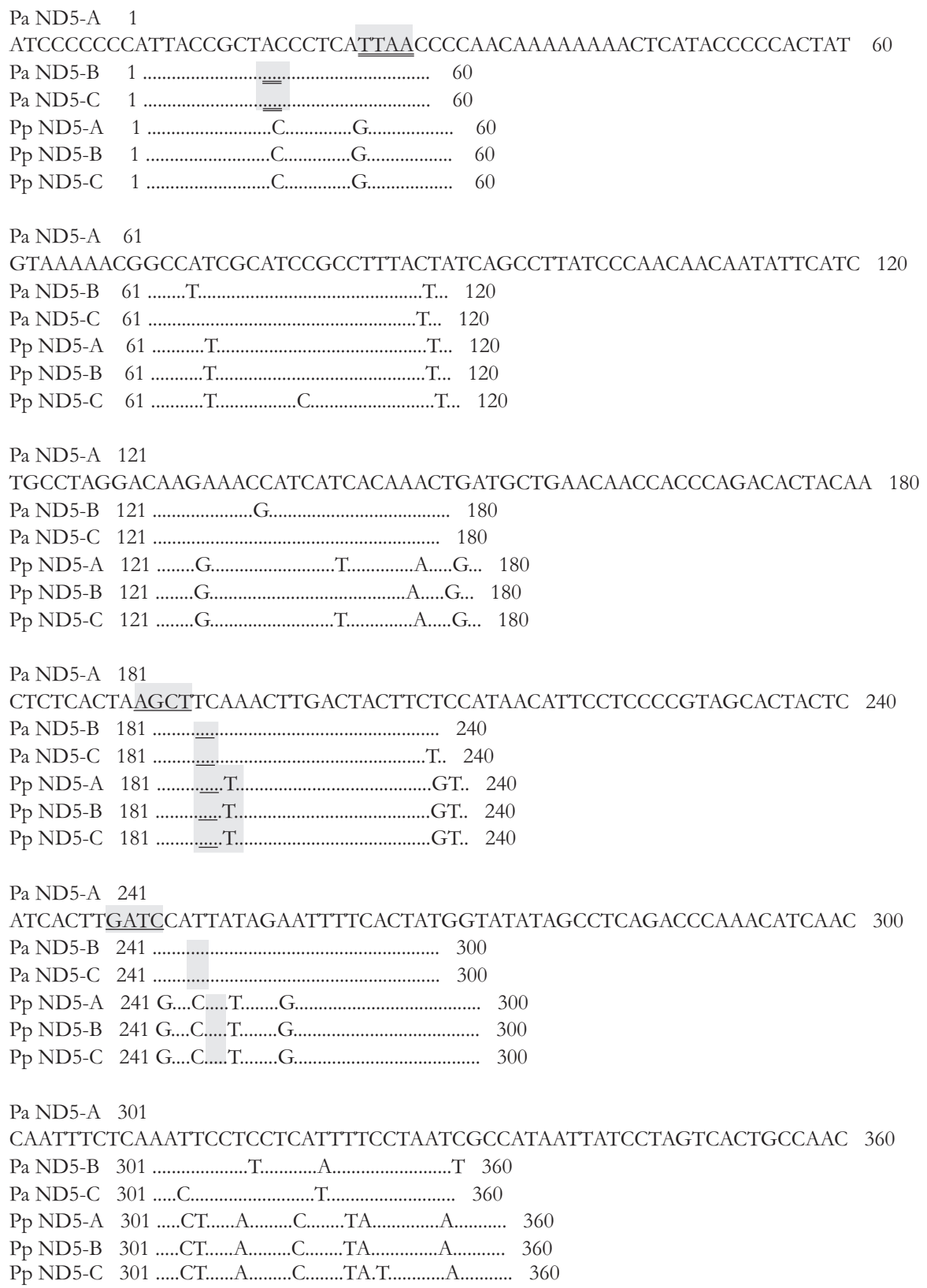


Table 2. Continued

Pa ND5-A 361

AACCTACTCCAACTCT'TCATCGGCTGAGAAGGCGTAGGAATCATATCCT'TCCTGCTCATT 420

Pa ND5-B 361 .................................................... 420

Pa ND5-C 361 ...................................................... 420

Pp ND5-A 361 .................................................. 420

Pp ND5-B 361 ................................................... 420

Pp ND5-C 361 .................................................... 420

Pa ND5-A 421

AGTTGATGATACGCCCGAACAGACGCTAACACAGCAGCTATTCAAGCAATCCTATACAAT 480

Pa ND5-B 421 ..................................................... 480

Pa ND5-C 421 ................................G........................... 480

Pp ND5-A 421 ........................................................ 480

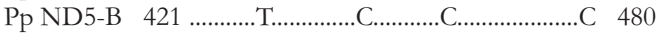

Pp ND5-C 421 ......................................................... 480

Pa ND5-A 481 CGT 483

Pa ND5-B $481 \ldots$.

Pa ND5-C $481 \ldots$.

Pp ND5-1 $481 \ldots$.

Pp ND5-3 $481 \ldots$.

Pp ND5-5 $481 \ldots \quad 483$

Table 3. Summary of digestion profiles with $A l u \mathrm{I}$ and $M s e \mathrm{I}$

\begin{tabular}{llc}
\hline \multirow{2}{*}{ mtDNA type } & \multicolumn{2}{c}{ Number of DNA fragments after digestion } \\
\cline { 2 - 3 } & \multicolumn{1}{c}{$A l u \mathrm{I}$} & Mse I \\
\hline Sumatra & 3 (ca. $50+220+270 \mathrm{bps})$ & 2 (ca. $50+490 \mathrm{bps})$ \\
\hline Borneo & 2 (ca. $220+320 \mathrm{bps})$ & 2 (ca. $220+320 \mathrm{bps})$ \\
\hline
\end{tabular}

As reported by Xu and Arnason (1996), the molecular differences between the two orangutans P. abelii and P. pygmaeus are considerably greater than those between species of hominoids (common/pygmy chimpanzee) and some other mammals (harbor/grey seals). Therefore, the two orangutans should be given the rank of separate species, $P$. abelii, Sumatra orangutan, and P. pygmaeus, Bornean orangutan. Analysis of molecular variation is commonly used in evaluation of animal populations for purposes of taxonomic. Furthermore the obtained result of Muir et al. (2000) based on mitochondrial DNA analyses suggested that mitochondrial lineage of Sumatran and Bornean orangutans has been isolated for an extended period of time. Hence, for practical uses, species discrimination of Orangutans is critical step to be applied to rehabilitation centers and zoos.

But, the new result revealed a rapid protocol to identify these two species by means of RFLP (Restriction Fragment Length Polymorphism) analyses of mtDNA (mitochondrial DNA). This technique can be applied easily to rehabilitation centres and zoos to resolve species discrimination problem there. Nonetheless Warren et al. (2001) found that four distinct subpopulations were identified in Borneo, as a result further study are required for assessment of molecular variation within Bornean orangutan using the same method. 


\section{CONCLUSION}

$A l u \mathrm{I}$ and $M s e \mathrm{I}$ could be used for species discrimination of Orangutans by means of PCR-RFLP analyses.

Applicability of the PCR-RFLP protocol reported here should be tested further in future study.

\section{ACKNOWLEDGMENT}

I would like to express my sincere gratitude for the DIPA BIOTROP 2008 research grant to support this research. Moreover, I am grateful for the laboratory assistance provided by the Laboratory of Biology and Reproduction, Primate Research Center and the staff of Animal Biosystematics and Ecology Division, Department of Biology, Bogor Agricultural University, Bogor. Several anonymous reviewers made numerous suggestions leading to considerable improvements in the manuscript.

\section{REFERENCES}

Groves C.P. 1971. Pongopygmaeus. Mammalian Species, 4: 1-6.

Janczewski D.N., Goldman D. and S.J. O'Brien. 1990. Molecular genetic divergence of orangutan (Pongo pygmaeus) subspecies based on isozyme and two-dimensional gel electrophoresis. Journal of Heredity, 81:375-387.

Muir C.C., Galdikas B.M.F. and A.T. Beckenbach. 1998. Is there sufficient evidence to elevate the orangutan of Borneo and Sumatra to separate species. Journal of Molecular Evolution, 51:471-480.

O'Brien S.J. 1994. Genetic and phylogenetic analyses of endangered species. Annual Review of Genetics, 28 : 467-489.

Rijksen H.D. and E. Meijaard. 1999. Our vanishing relative; the status of wild orang-utans at the close of the twentieth century. Kluwer Academic Publishers, Dordrecht, the Netherlands.

Uchida A. 1996. What we don't know about great ape variation. Trends in Ecology \& Evolution, 11: 163-168.

Warren K.S., Verschoor E.J., Langenhuijen S., Heriyanto, Swan R.A., Vigilant L. and J.L. Heeney. 2001. Speciation and Intrasubspecific variation of Bornean Orangutan, Pongo pygmaeus pygmaeus. Molecular Biology and Evolution, 18: 472-480.

Xu X. and U. Arnason. 1996. The mitochondrial DNA molecule of Sumatran and a molecular proposal for two (Bornean and Sumatra) species of orangutan. Journal of Molecular Evolution, 43: 431-437.

Zang Y., Ryder O.A. and Y. Zhang. 2001. Genetic divergence of orangutan subspecies (Pongo pygmaeus). Journal of Molecular Evolution, 52: 516-526.

Zhi L., Karesh W.B., Kanczewski D.N., Frazier-Taylor H., Sajuthi D., Gombek F., Andau M., Martenson J.S. and S.J. O'Brien. 1996. Genomic differentiation among natural populations of orang-utan (Pongopygmaeus). Current Biology, 6: 1326-1336. 